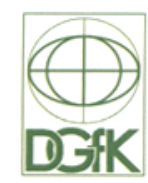

\title{
Deutscher Kartographentag
}

\author{
15. bis 17.9.2015 \\ Messegelände Stuttgart \\ im Rahmen der INTERGEO ${ }^{\circledR}$
}

Lösungen

für Big und Open Data

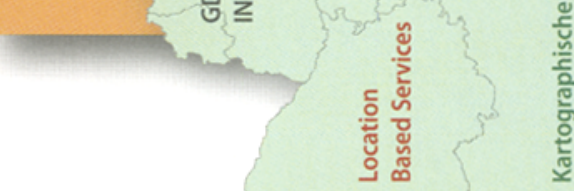

\section{Dienstag, 15. September}

10:00 .. 11:30 Uhr

\section{Eröffnung}

The Age of the Location Platform: How Mapping and GIS are Transforming the Work Environment Chris Cappelli (ESRI)

The Future of the Map - the Map of the Future Georg Gartner (TU Wien, ICA)

13:00 ..14:30 Uhr

AdV-Projekt Generalisierung

Motivation, Ziele und Strategien für die Implemen. tierungspartnerschaft ATKIS-Generalisierung Hansgerd Terlinden (Hessisches Landesamt für Bodenmanagement und Geoinformation, Wiesbaden)

Automatische Generalisierungsprozesse für die Ableitung von AAA-Produkten

Dietrich Schürer (Landesamt für Vermessung und Geobasisinformation Rheinland-Pfalz, Koblenz)

Die Modellgeneralisierung am Beispiel des Übergangs vom ATKIS-Basis-DLM zum ATKIS-DLM5O

Bernd Sender (Bezirksregierung Köln)

Die Automatische Kartographische Generalisierung für die Herstellung des amtlichen Kartenwerkes 1:50.000 (DTK50)

Dietmar Gehrmann (Landesamt für Vermessung und Geobasisinformation, Koblenz)

\section{5:00 .. 16:00 Uhr}

\section{Kartographische Produktion}

swissnames3D - die neue Datenbank für geografische Namen von swisstopo

Stefan Neudeck (Bundesamt für Landestopographie swisstopo)

Automatische Gebäudegeneralisierung mit ArcGIS: durchgeführt am Beispiel der Schweizer Landeskarte 1:50'000

Anna Vetter (Esri Schweiz AG)

Basispläne/Stadtpläne aus AAA-Daten - ein Praxisbericht

Ajay Mathur (Axes Systems AG)
14:15 .. 16:15 Uhr

\section{Mitgliederversammlung}

Die Versammlung findet im Flughafen Stuttgart, Terminal 1, Ebene 4, Raum „Klemm“ statt (Fußweg zum Messegelände ca. 5 Minuten).

Weitere Informationen erhalten Mitglieder in einer gesonderten Einladung.

\section{Donnerstag, 17. September}

\section{9:30 .. 10:30 Uhr \\ Urbane Anwendungen}

300 Jahre Karlsruhe: 1715 - 1834 - 1915 - 1945 - 2015. Digitales historisches 3D-Stadtmodell

Detlef Günther-Diringer (Hochschule Karlsruhe)

Kartographische Darstellung von Lärm - Von auditiver Wahrnehmung zu visueller Darstellung Beate Weninger (HafenCity Universität Hamburg)

Lärmpausen am Flughafen Frankfurt - Komplexe Ideen mit GIS aufbereiten, analysieren und bewerten Günter Lanz (Gemeinnützige Umwelthaus AG) \& Wassiolios Kazakos (disy Informationssystem $\mathrm{GmbH}$ )

\section{$11: 00$.. 12:00 Uhr}

\section{Kartographische Visualisierungen}

Einsatz von kartographischen Visualisierungen auf Webseiten: Erfahrungen am Beispiel des OnlineAuftritts zur Buchreihe "Landschaften in Deutschland" Jana Moser \& Sebastian Koslitz (Institut für Länderkunde, Leipzig)

Datengetriebene Erkennung und Untersuchung kurzfristiger Reaktionen in der Kraftstoffpreisgestaltung benachbarter Tankstellen

Johannes Kröger (HafenCity Universität Hamburg)

Highly Interactive, Beautiful Maps for Exploring Spatial Data: Why Design-driven Cartography Looks Different

Frank Heidmann, Till Nagel \& Sebastian Meier (FH Potsdam)
13:00 .. 14:00 Uhr

Gebrauchstauglichkeit kartographischer Darstellungen

Eine empirische Studie zu Effekten von Grünblindheit (Deuteranopie) auf das kartenbasierte Positionsgedächtnis

Dennis Edler, Sirle Edler \& Frank Dickmann (Ruhr-Universität Bochum)

Untersuchung landmarkenbasierter Navigationsprozesse von Fußgängern mithilfe eines mobilen Eye-Tracking-Systems

Conrad Franke, Lotta Atzenbeck \& Jürgen Schweikart (Beuth Hochschule, Berlin)

Zum Problem der modellhaft adäquaten Abbildung der Landschaft in topographischen Manuskriptkarten des 18. Jahrhunderts und heutigen topographischen Karten

Beata Medynska-Gulij (Adam Mickiewicz Universität, Poznan, Polen)

\section{4:30.. 15:30 Uhr}

\section{Aktuelle Forschungsthemen}

Generalisierung ist nicht nur ein Maßstabsproblem: Überlegungen zur Ableitung medienübergreifender Geometrien auf der Grundlage offener Basisdaten Francis Harvey, Eric Losang, Jana Moser \& Thomas Zimmermann (Institut für Länderkunde, Leipzig)

Aktuelle Fortschritte in der automatischen Schriftplatzierung

Andreas Reimer \& Maxim Rylov (Universität Heidelberg)

Die Renaissance weißer Flecken: Kartographische Visualisierung von Forschungsdaten aus der historischen Klimatologie

Sebastian Specht et al. (Institut für Länderkunde, Leipzig)

\begin{tabular}{lrrr} 
Anmeldung \& & Preise \\
\hline & $\begin{array}{c}\text { Dauerkarte } \\
\text { Buchung } \\
\text { bis } 31.7 .\end{array}$ & $\begin{array}{c}\text { Buchung } \\
\text { ab 1.8. }\end{array}$ & $\begin{array}{c}\text { Tages- } \\
\text { karte }\end{array}$ \\
\hline Normalpreis & $285 €$ & $335 €$ & $130 €$ \\
$\begin{array}{l}\text { DGfK-Mitglieder } \\
\begin{array}{l}\text { DGfK-Mitglieder } \\
\text { in Ausbildung }\end{array}\end{array}$ & $235 €$ & $275 €$ & $110 €$ \\
\hline
\end{tabular}

